\title{
Hepatic Arterial Embolization versus Chemoembolization in the Treatment of Liver Metastases from Well-Differentiated Midgut Endocrine Tumors: A Prospective Randomized Study
}

\author{
Frédérique Maire ${ }^{\mathrm{a}}$ Catherine Lombard-Bohas ${ }^{\mathrm{d}}$ Dermot O'Toole ${ }^{\mathrm{a}}$ \\ Marie-Pierre Vullierme ${ }^{b}$ Vinciane Rebours $^{a}$ Anne Couvelard ${ }^{c}$ \\ Anne Laure Pelletier ${ }^{a}$ Magaly Zappa ${ }^{b}$ Frank Pilleul $^{\mathrm{e}}$ Olivia Hentic $^{a}$ \\ Pascal Hammel ${ }^{\mathrm{a}}$ Philippe Ruszniewski ${ }^{\mathrm{a}}$ \\ Services de a Gastroentérologie-Pancréatologie, ${ }^{b}$ Radiologie, et ${ }^{c}$ Anatomo-Pathologie, Hôpital Beaujon, AP-HP, Clichy, \\ Services de ${ }^{\mathrm{d}}$ Oncologie médicale, et ${ }^{\mathrm{e}}$ Radiologie, CHU Edouard Herriot, Lyon, France
}

\section{Key Words \\ Chemoembolization - Embolization • Liver metastases • \\ Midgut endocrine tumors $\cdot$ Progression-free survival}

\begin{abstract}
Background: Liver surgery is the best treatment for endocrine liver metastases, but it is often impossible due to diffuse disease. Systemic chemotherapy is poorly effective. Hepatic arterial embolization (HAE) and chemoembolization (HACE) have shown efficacy but have never been compared. Patients and Methods: Patients with progressive unresectable liver metastases from midgut endocrine tumors were randomly assigned to receive HAE or HACE (two procedures at 3-month interval). The primary end point was the 2-year progression-free survival (PFS) rate. Secondary end points were response rates, overall survival, and safety. Results: Twelve patients were assigned to receive HACE and 14 to receive HAE. The patient characteristics were well matched across the treatment arms. The 2-year PFS rates were 38 and $44 \%$ in the HACE and HAE arms, respectively $(p=0.90)$. Age,
\end{abstract}

gender, previous resection of the primary tumor or liver metastases, extent of liver involvement, and concomitant treatment with somatostatin analogues were not associated with changes in PFS, whereas elevated baseline urinary 5-HIAA and serum chromogranin A levels were associated with shorter PFS. The 2-year overall survival rates were 80 and $100 \%$ in the HACE and HAE arms, respectively $(p=0.16)$. The disease control rate on CT scan was $95 \%$. Grade 3 toxicity occurred in $19 \%$ of patients, with no treatment-related deaths and no differences in the treatment arms. Conclusion: HACE and HAE are safe and permit tumor control in $95 \%$ of patients with progressive liver metastases from midgut endocrine tumors. The 2-year PFS was not higher among patients receiving $\mathrm{HACE}$, not favoring the hypothesis of an additive efficacy of arterial chemotherapy or embolization alone.

Copyright $\odot 2012$ S. Karger AG, Basel

This study was presented at the 2011 ENETS annual meeting in Lisbon, Portugal.

\section{KARGER}

Fax +4161306 1234

E-Mail karger@karger.ch

www.karger.com
C 2012 S. Karger AG, Basel

0028-3835/12/0964-0294\$38.00/0

Accessible online at:

www.karger.com/nen
Dr. Frédérique Maire

Service de Gastroentérologie-Pancréatologie, Hôpital Beaujon, AP-HP

100 , Boulevard du Général Leclerc

FR-92118 Clichy Cedex (France)

Tel. +33 1408752 25, E-Mail frederique.maire@bjn.aphp.fr 


\section{Introduction}

Liver metastases are frequent in patients with digestive endocrine tumors and significantly influence overall prognosis $[1,2]$. Liver surgery is considered as the treatment of choice, with 5 -year survival rates $>70 \%$ in patients amenable to resection [3-5]. However, liver surgery is frequently impossible due to the extent of the disease. Various surgical approaches have been proposed to these patients, such as a two-step surgical procedure or a combination of surgery and radiofrequency ablation [5-8]. Even with the development of these techniques, the resection of more than 10 or 20 bilateral lesions is usually considered as unfeasible. Liver transplantation can be proposed to very selected patients. Systemic chemotherapy in patients with diffuse and/or progressive liver metastases yields disappointing results in patients with metastases of midgut origin $[9,10]$. In addition, it has been recently demonstrated that somatostatin analogues can lengthen time to tumor progression, but are less effective in patients with high hepatic tumor load [11]. Other treatment options include interferon, peptide receptor radionuclide therapy and new target therapies $[12,13]$.

Locoregional strategies such as vascular occlusion inducing ischemia in these highly vascularized tumors have been proposed using either surgical or radiological techniques (e.g. surgical ligation of the hepatic artery, transient hepatic ischemia, or sequential hepatic dearterialization). In 1994, Moertel et al. [9] found that patients treated with a combination of hepatic arterial occlusion and systemic chemotherapy had a higher response rate than those treated with arterial occlusion alone. Because hepatic metastases derive most of their blood supply from the hepatic artery, regional delivery of chemotherapy offers pharmacokinetic advantages over systemic administration. The rationale of chemoembolization (HACE) is to combine the effects of chemotherapy injected in the hepatic artery with those of anoxia induced by embolization. The association of small particles of iodized oil containing doxorubicin obliterates the peripheral arterial branches and then the tumor vessels, whereas gelatin sponge particles embolize proximal feeding arteries. The combination of these therapeutic methods may increase intratumoral drug concentration and dwelling time in cancer cells [14]. Moreover, certain drugs are more efficient on anoxic cells [15]. Trans-catheter arterial HACE and embolization are effective in controlling hormonal symptoms and reducing tumor size [14, 16-19]. The additive efficacy of intra-arterial chemotherapy as compared with embolization has not been proven to date.
The aim of the present study was to compare the efficacy of hepatic arterial embolization (HAE) and HACE on the progression-free survival (PFS) rates in patients with endocrine liver metastases of midgut origin, in a prospective randomized trial.

\section{Patients and Methods}

\section{Study Design and Patients}

This study was a prospective randomized trial conducted in two French centers (Paris and Lyon) from 2002 through 2008. The institutional review board at each center approved the protocol, and all patients provided written informed consent. Eligible patients were at least 18 years of age and had histologically confirmed endocrine liver metastases from midgut tumors [20]. Ki67 scoring method was also evaluated by the same pathologist. Ki-67 protein was detected with the murine monoclonal antibody MIB-1 (Dako Denmark, Glostrup, Denmark).

Liver metastases were considered unresectable in all patients. The primary tumor was removed whenever possible. Extrahepatic metastases were considered as exclusion criteria, with the exception of lymph node involvement. Patients had to present with either progressive liver metastases within 12 months before the inclusion, i.e. progression of $>25 \%$ between two consecutive imaging procedures, or liver tumoral involvement of $>50 \%$ as judged on CT scan. If present, carcinoid syndrome had to be controlled by somatostatin analogues.

Patients with liver dysfunction (albumin $<2.8 \mathrm{mg} / \mathrm{dl}$, bilirubin $>2 \mathrm{mg} / \mathrm{dl}$, prothrombin rate $<70 \%$, aspartate aminotransferase $>100 \mathrm{IU} / \mathrm{l}$, ascites), renal dysfunction (creatinine clearance $<50$ $\mathrm{ml} / \mathrm{min}$ ), history of HAE or HACE, sus-hepatic or portal vein thrombosis, cardiac insufficiency, instable coronary disease, heart stroke within the previous 3 months, uncontrolled hyperthyroidism, Karnofsky index $<70 \%$, contrast allergy as well as pregnant, breast-feeding or fertile women without contraceptive use were not eligible.

\section{Randomization}

Patients were randomly assigned to receive hepatic intra-arterial embolization or HACE. Randomization was performed centrally with the use of an adaptive randomization procedure stratified per center and progression group, defined as $>25 \%$ within 3 months, $>25 \%$ within 3-12 months, or liver involvement of $>50 \%$ in patients. Treatment was administered after randomization and was repeated 3 months thereafter. Intravenous hydration was started $2 \mathrm{~h}$ before treatment (normal saline 2,000 $\mathrm{ml} /$ day) and patients were premedicated with antibiotics. Patients with carcinoid syndrome were administered octreotide $200 \mu \mathrm{g}$ subcutaneously before the procedure and every $8 \mathrm{~h}$ afterward during $48 \mathrm{~h}$ to prevent a carcinoid crisis.

HACE and HAE were performed by a transfemoral approach by experienced interventional radiologists. In the HACE arm, the cytotoxic drug was doxorubicin $\left(50 \mathrm{mg} / \mathrm{m}^{2}\right)$ dissolved in normal saline and combined with $10-15 \mathrm{ml}$ of iodized oil (Lipiodol Ultra Fluid; Guerbet, Aulnay-sous-Bois, France), and injected into the branches of the hepatic artery distal to the gastroduodenal artery, followed by embolization with gelatin sponge particles (Gel- 
itaspon; Gelita Medical, Amsterdam, The Netherlands) until a marked decrease in blood flow was observed. The HAE arm received only gelatin sponge particle embolization with the same procedure. Whenever possible, both lobes of the liver were subjected to embolization or HACE during each session. The procedure was performed under local anesthesia. Patients were closely monitored hemodynamically during the procedure. They were hospitalized for at least 3 days, depending on the severity of postembolization syndrome or any complications. Antibiotics were administered during $48 \mathrm{~h}$ after the procedure.

Follow-Up

Baseline assessment included medical history, physical examination with evaluation of carcinoid symptoms, biological analyses performed within 7 days before treatment initiation, and tumor evaluation (using CT scan or MRI) performed within 28 days of the start of treatment.

During the treatment period, physical examination, pain assessment using a visual analogical scale, and analgesic intake and toxicity were recorded after 3, 6, 12, 18 and 24 months. Tumor assessment by the same imaging method throughout the follow-up period was required in both arms after 3, 6, 12, 18 and 24 months or earlier if clinically indicated. Tumor response was measured according to the World Health Organization criteria. A complete response was defined as the complete disappearance of all recognizable tumor in the liver, a partial response was defined as a reduction of $>50 \%$ in tumor size, a minor response was defined as a reduction of $<50 \%$ but of $>25 \%$ in tumor size, stable disease was defined as a reduction or progression of disease of $<25 \%$, and progressive disease was defined as an increase of $>25 \%$ in tumor size over that measured at the time of maximal tumor shrinkage or as the development of new lesions. The size of the liver metastases was determined by multiplying the longest dimension of the tumor mass by its greatest perpendicular dimension, as measured on CT or MRI studies. A biological response was assessed by a decrease of $>25 \%$ of levels of the serum chromogranin A. Toxicity was graded according to the National Cancer Institute Common Toxicity Criteria version 2.

\section{Primary and Secondary End Points}

The primary end point was the PFS rate, calculated from the day of randomization until death or evidence of tumor progression as assessed by CT scan measurement (progression of $>25 \%$ of liver metastases and/or development of new liver metastases). Although treatment was directed only on liver metastases, the occurrence of extrahepatic metastases reflecting disease progression was considered as a treatment failure.

Secondary end points were side effects, morphological and biological response rates and overall survival (OS) rates.

\section{Statistical Analysis}

A sample size calculation was performed, assuming from historical data a 2 -year PFS of $50 \%$ in the embolization arm. To demonstrate an increase in this rate to $75 \%$ in the HACE arm, a total of 68 patients was required, using a log-rank test at the two-sided 0.05 level with a power of $90 \%$. The recruitment period was 2 years, and all patients were followed up for 24 months after inclusion. Due to the insufficient number of included patients within 2 years, an amendment was made to increase the inclusion period to 2 supplementary years.
Log-rank tests and Kaplan-Meier estimations were performed for the analysis of both PFS and OS. $\chi^{2}$ or Fisher's exact tests, when suitable, were used to compare qualitative data. Differences were assumed to be significant at $\mathrm{p}<0.05$. Cox proportional hazards modeling was used to examine the effect of various prognostic factors having a $\mathrm{p}$ value $<0.25$ in univariate analysis.

\section{Results}

\section{Patient Characteristics}

Twenty-six patients, median age of 58 years (range 3879) were included in the present study. All patients had a well-differentiated midgut endocrine tumor. The Ki-67 index was available in 22 patients and was $\leq 2 \%, 3-5 \%$ and $6-10 \%$ in 16, 5 and 1 patient, respectively. Twentytwo patients (85\%) had previously undergone resection of the primary tumor. In the 4 remaining patients, the primary tumor was unknown after an extensive work-up. Four patients (15\%) had previously undergone surgical resection of liver metastases. Nineteen patients (73\%) presented with a carcinoid syndrome, all of them controlled by somatostatin analogues. Two patients (8\%) had tricuspid valve disease in relation with the carcinoid syndrome. The median Karnofsky index was 90 (range 70$100)$. Liver involvement was $<25 \%, 25-50 \%$ and $>50 \%$ in $13(50 \%), 8(31 \%)$ and $5(19 \%)$ patients, respectively. Median time between the diagnosis of the endocrine tumor and inclusion in the present study was 22 months (range $3-137)$. At time of inclusion, 15 patients (58\%) had progressive disease in more than 3 months, 8 patients $(31 \%)$ had progressive disease in less than 3 months, and 3 patients (11\%) had no measurable progression but liver involvement of $>50 \%$. Baseline serum chromogranin A levels were available in 24 patients and were above the normal value in 18 (75\%). The median serum chromogranin A level was $155 \mathrm{mmol} / \mathrm{l}$ (range 50-23,034). Baseline urinary 5-HIAA levels were available in 24 patients and were above the normal in 12 (50\%). Median urinary 5-HIAA level was $189 \mu \mathrm{mol} / 24 \mathrm{~h}$ (range 24-1,711). High levels of urinary 5-HIAA were observed in 42 and $17 \%$ of patients with high or low liver involvement, respectively.

\section{Treatment and Follow-Up}

Twelve and 14 patients were randomly assigned to receive HACE or embolization, respectively. The baseline patient characteristics were well matched across the treatment arms (table 1). All patients except 3 received the two sessions of treatment as planned. The median follow-up was 16.9 months (range 1-28.5), and was not different between both arms. 
Fig. 1. Kaplan-Meier curves showing PFS in patients with liver metastases from midgut endocrine tumors treated with HACE or bland embolization.
Number of patients $\begin{array}{lllllll}\text { Chemoembolization } & 12 & 9 & 7 & 3 & 3 & 2\end{array}$ Embolization

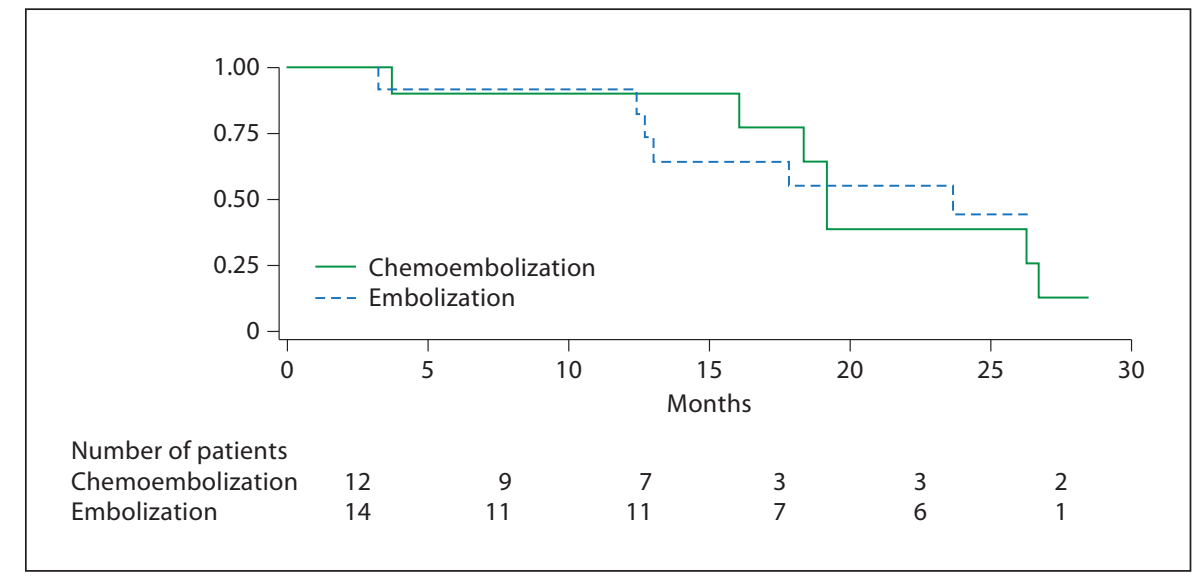

Table 1. Patient profile according to treatment with hepatic intra-arterial chemoembolization or embolization

\begin{tabular}{|c|c|c|c|}
\hline & $\begin{array}{l}\text { Chemoembolization } \\
(\mathrm{n}=12)\end{array}$ & $\begin{array}{l}\text { Embolization } \\
(\mathrm{n}=14)\end{array}$ & $\mathrm{p}$ \\
\hline Gender & & & 0.07 \\
\hline Male & 7 & 9 & \\
\hline Female & 5 & 5 & \\
\hline Median age & $65(38-71)$ & $56(41-79)$ & 0.53 \\
\hline Resection of the primary tumor & 10 & 12 & 1 \\
\hline Previous resection of liver metastases & 2 & 2 & 1 \\
\hline Carcinoid syndrome & 8 & 11 & 0.6 \\
\hline Concomitant treatment with somatostatin analogues & 8 & 11 & 0.66 \\
\hline Median urinary 5-HIAA, $\mu \mathrm{mol} /$ day & 192 & 94 & 0.46 \\
\hline Median serum chromogranin A, mmol/l & 135 & 192 & 0.20 \\
\hline Body mass index & 24 & 23 & 0.97 \\
\hline Liver involvement & & & 1 \\
\hline$<25 \%$ & 7 & 6 & \\
\hline $25-50 \%$ & 3 & 5 & \\
\hline$>50 \%$ & 2 & 3 & \\
\hline Evolutivity & & & 1 \\
\hline Liver involvement of $>50 \%$ and no evaluable progression & 1 & 2 & \\
\hline Progression of $>25 \%$ in less than 3 months & 4 & 4 & \\
\hline Progression of $>25 \%$ in more than 3 months & 7 & 8 & \\
\hline Median follow-up, months & 17.2 & 15.4 & 0.83 \\
\hline Disease progression & 7 & 6 & 0.43 \\
\hline
\end{tabular}

Figures in parentheses are ranges.

\section{Primary End Point}

The 1-year PFS rates were 90.0 and $91.6 \%$ in the HACE and HAE arms, respectively. The 2-year PFS rates were 38.5 and $44.0 \%$ in the HACE and HAE arms, respectively $(\mathrm{p}=0.90)$ (fig. 1$)$. The median PFS was 19.2 months (range 16.1-26.8) in the HACE arm and 23.6 months (range 12.7-n.a.) in the HAE arm.

HAE versus HACE in the Treatment of Liver Metastases from Midgut Tumors
Age, gender, BMI, previous resection of the primary tumor or liver metastases, extent of liver involvement, presence of carcinoid syndrome, concomitant treatment with somatostatin analogues, duration of the disease before inclusion and previous tumor progression were not associated with changes in PFS at univariate analysis. Elevated baseline urinary 5-HIAA levels were significantly associated with shorter PFS (2-year PFS rates were 55 and 
$16 \%$ in patients with normal and elevated baseline urinary 5 -HIAA levels, respectively, $p=0.03$ ). There was a trend in correlation between baseline serum chromogranin A levels and 2-year PFS rates (67 and 30\% in patients with normal and elevated baseline chromogranin A levels, respectively, $\mathrm{p}=0.07$ ). At multivariate analysis, normal baseline urinary 5-HIAA levels were associated with delayed progression (OR 5.4, 95\% CI 1.2-24.6, $\mathrm{p}=0.02$ ).

\section{Secondary End Points}

Study discontinuation was required in 15 patients $(58 \%)$ due to progression $(\mathrm{n}=9)$, death $(\mathrm{n}=2)$, adverse events $(n=2)$, or patient decision $(n=2)$. Thirteen patients $(50 \%)$ presented with tumoral progression during the 24-month follow-up. Two patients (8\%) died during the follow-up period. One patient died of tumor progression 17 months after inclusion. The other patient had a history of coronary disease. He presented a myocardial ischemia 5 days after HACE, with altered left ventricular function and pulmonary edema. The coronarography showed IVA stenosis requiring coronary stent. Secondarily, he presented acute renal failure, which was attributed to initial hypovolemia due to cardiac complication and iodine injection during coronarography.

The 1-year OS rates were 91.6 and $100.0 \%$ in the HACE and $\mathrm{HAE}$ arms, respectively, and the 2-year OS rates were 80.2 and $100.0 \%$ in the HACE and HAE arms, respectively $(\mathrm{p}=0.16)$. Twenty-three patients were evaluable for morphological response after the two treatment sessions. Partial and minor response, stable disease and progression were observed in 7 (30\%), 8 (35\%), 7 (30\%) and $1(5 \%)$ patient, respectively. No factor was significantly associated with tumor response. The control of tumor progression (partial response + minor response + stable disease) was observed in 22 patients (95\%): 100 and $92 \%$ in the HACE and HAE arms, respectively $(\mathrm{p}=0.74)$.

Biological response was evaluable in 20 patients. A decrease of $>25 \%$ of the serum chromogranin A level was observed in 15 patients (75\%): 67 and $82 \%$ in the HACE and HAE arms, respectively $(\mathrm{p}=0.69)$. Serum chromogranin A levels were stable in 3 patients (15\%) and increased in 2 patients (10\%). Biological control (decreased + stable levels) was noted in 18 patients (90\%): 89 and 91\% in the HACE and HAE arms, respectively $(\mathrm{p}=0.77)$. There was a trend for correlation between biological response and morphological tumor response $(\mathrm{p}=0.06)$.

\section{Adverse Events (table 2)}

Adverse events occurred in 23 patients (88\%), with grade 3 toxicity in 5 patients (19\%). The most common
Table 2. Adverse events

$$
\begin{array}{ll}
\text { HACE } & \text { HAE } \\
(n=12) & (n=14)
\end{array}
$$

\begin{tabular}{lrcl}
\hline Treatment-related death & 0 & 0 & - \\
Adverse events & & & \\
$\quad$ Postembolization syndrome & 10 & 10 & \\
$\quad$ Carcinoid crisis & 2 & 0 & \\
$\quad$ Acute liver failure & 1 & 2 & 0.30 \\
$\quad$ Neutropenia & 2 & 0 & \\
Total adverse events & 11 & 12 & 2 \\
$\quad$ Any grade & $3^{*}$ & $2^{* *}$ & \\
$\quad$ Grade 3 &
\end{tabular}

* Neutropenia, $\mathrm{n}=2$; acute liver failure, $\mathrm{n}=1$.

** Acute liver failure, $\mathrm{n}=2$.

side effect was postembolization syndrome with vomiting, abdominal pain and fever occurring in 20 patients (77\%). Recovery was complete in all patients. Carcinoid crisis occurred in 2 patients despite octreotide prophylaxis controlled with intravenous somatostatin analogues administration. Three episodes of transient acute liver failure with complete recovery were noted. The incidence of complications was similar in both arms $(\mathrm{p}=0.30)$.

\section{Discussion}

HAE and HACE are accepted treatment options for patients with unresectable liver metastases from endocrine tumors. To date, there is no definite survival advantage of one treatment technique over the other. Local administration of chemotherapy would theoretically add an antitumoral effect of embolization of arterial flow in endocrine liver metastases. The present study is the first randomized prospective trial which compares both.

Numerous studies have shown that HAE and HACE are effective in controlling hormonal symptoms and reducing tumor size $[14,15,17,19,21-26]$. The tumor response rates are variable in the literature (33-92\%) [14, 17-19]. These results have to be interpreted cautiously for several reasons. Most studies included a small number of patients. Tumor types could be heterogeneous, including not only endocrine tumors but also hepatocellular carcinoma, GIST or melanoma $[25,27]$. Moreover, endocrine liver metastases could arise from various midgut or foregut primaries $[19,23,24]$. Disease progression at time of inclusion was rarely required, and most of the patients were included in presence of hormonal symptoms. PFS 
could thus not be assessed. Finally, the techniques varied concerning cytotoxic agent, number and interval between sessions. In the present study, all patients had liver metastases of midgut origin with histological confirmation of the endocrine well-differentiated type. All patients had progressive tumors at time of inclusion, except those with major liver involvement in whom treatment was readily required. The embolization technique was standardized, with or without local additive chemotherapy depending on the randomization arm. The concomitant use of other medications was carefully reported to exclude confounding variables, in particular somatostatin analogues which can have antiproliferative effects. Baseline patient characteristics were well matched across the treatment arms.

Our primary end point was the PFS rate. We found no differences in the 2-year PFS rates in patients who received HACE as compared with HAE (38 and 44\%, respectively, $\mathrm{p}=0.90$ ). The median PFS was 19.2 and 23.6 months in the HACE and HAE arms, respectively, which is a favorable result compared with other treatment modalities. Two factors were associated with higher PFS rates: low baseline urinary 5-HIAA and serum chromogranin A levels ( $p=0.03$ and $p=0.07$, respectively). The role of baseline urinary 5-HIAA levels was confirmed at multivariate analysis $(\mathrm{p}=0.02)$. In the literature, few studies analyzed PFS due to the methodological limits already discussed. In a non-randomized study, Gupta et al. [19] reported that the addition of intra-arterial chemotherapy to embolization did not improve the PFS rates.

In the present study, the morphological disease control rate was $95 \%$, similar to that previously reported (7796\%) $[17-19,23-25,27,28]$. We did not identify any predictive factors of tumor response. The tumor response rates were similar in both arms. The biological disease control rate was $90 \%$, without any difference between both arms. The functional response, defined as the control of symptoms, could not be assessed by the present study because carcinoid syndrome control was required to be eligible for inclusion.

The 2 -year OS rates were 80 and $100 \%$ in the HACE and HAE arms, respectively $(\mathrm{p}=0.16)$. The median OS was not evaluable and the analysis of predictive factors of OS was not relevant due to the small number of deaths. In the literature, the 2-year OS rates after treatment of midgut endocrine liver metastases were $67-80 \%$, with a median OS near 40 months (range 31-80) $[19,24,26]$. The percentages of changes in urinary 5-HIAA and serum chromogranin A levels were correlated with prolonged survival in one study and have been proposed to be used as predictors of long-term outcome [26].

HAE versus HACE in the Treatment of Liver Metastases from Midgut Tumors
In the present study, we have chosen doxorubicin as cytotoxic agent in the HACE arm due to its better tolerance as compared with streptozotocin, and not requiring general anesthesia for the procedure. At the time of study initiation, no data showing differences in efficacy according to the cytotoxic agent used were available $[19,29]$. One recent study, including patients with pancreatic and midgut primaries, suggested higher response rates with streptozotocin. Nevertheless, as treatment assignment was not randomized, it was not possible to conclude that streptozotocin is more efficient than doxorubicin [15].

Most patients experienced some degree of postembolization syndrome, which completely resolved within 48 $72 \mathrm{~h}$. Major side effects occurred in less than $20 \%$ of patients in all the series in the literature, as in our study (19\%). We did not find a higher incidence of complications in the HACE versus HAE arms $(\mathrm{p}=0.30)$. Although both techniques have never been compared, the compilation of results from various studies indicates their safety $[19,30]$.

The main limitation of the present study is the number of patients, as the calculated sample was not reached due to recruitment problems. We cannot exclude that the resulting lack of power could mask any differences in PFS between both arms of treatment. However, the homogeneity of the patient population, the fact that midgut endocrine tumors are usually considered as chemoresistant (in part due to their low proliferative index), and the lack of any trend in longer PFS in patients receiving HACE as compared to HAE strengthen the validity of our results. Using drug-eluting beads with HACE could also add efficacy as suggested in endocrine liver metastases from pancreatic primaries [31] and warrants evaluation in patients with midgut tumors.

In conclusion, our study suggests that hepatic arterial (chemo)embolization is a safe and very effective treatment for patients with progressive liver metastases and/ or major liver involvement from midgut endocrine tumors. Although the number of patients was limited, our current results suggest that the addition of intra-arterial chemotherapy to embolization does not prolong PFS.

\section{Acknowledgment}

The authors thank Novartis Pharma for the financial grant that contributed to conducting the study.

\section{Disclosure Statement}

The authors declare that there is no conflict of interest that could be perceived as prejudicing the impartiality of the research reported. 


\section{References}

1 Plockinger U, Rindi G, Arnold R, et al: Guidelines for the diagnosis and treatment of neuroendocrine gastrointestinal tumours. A consensus statement on behalf of the European Neuroendocrine Tumour Society (ENETS). Neuroendocrinology 2004;80: 394-424.

-2 Hentic O, Couvelard A, Rebours V, et al: Ki67 index, tumor differentiation, and extent of liver involvement are independent prognostic factors in patients with liver metastases of digestive endocrine carcinomas. Endocr Relat Cancer Cancer 2010;18:51-59.

3 Norton JA, Warren RS, Kelly MG, et al: Aggressive surgery for metastatic liver neuroendocrine tumors. Surgery 2003;134:10571063.

-4 Sarmiento JM, Heywood G, Rubin J, et al: Surgical treatment of neuroendocrine metastases to the liver: a plea for resection to increase survival. J Am Coll Surg 2003;197: 29-37.

5 Kianmanesh R, Sauvanet A, Hentic O, et al: Two-step surgery for synchronous bilobar liver metastases from digestive endocrine tumors: a safe approach for radical resection. Ann Surg 2008;247:659-665.

6 Jaeck D, Oussoultzoglou E, Rosso E, et al: A two-stage hepatectomy procedure combined with portal vein embolization to achieve curative resection for initially unresectable multiple and bilobar colorectal liver metastases. Ann Surg 2004;240:1037-1049.

7 Elias D, Goéré D, Leroux G, et al: Combined liver surgery and RFA for patients with gastroenteropancreatic endocrine tumors presenting with more than 15 metastases to the liver. Eur J Surg Oncol 2009;35:1092-1097.

8 O'Toole D, Maire F, Ruszniewski P: Ablative therapies for liver metastases of digestive endocrine tumours. Endocr Relat Cancer 2003, 10:463-468.

-9 Moertel CG, Johnson CM, McKusick MA, et al: The management of patients with advanced carcinoid tumors and islet cell carcinomas. Ann Intern Med 1994;120:302-309.

10 Engstrom PF, Lavin PT, Moertel CG, et al: Streptozocin plus fluorouracil versus doxorubicin therapy for metastatic carcinoid tumor. J Clin Oncol 1984;2:1255-1259.

11 Rinke A, Müller HH, Schade-Brittinger C, et al: Placebo-controlled, double-blind, prospective, randomized study on the effect of octreotide LAR in the control of tumor growth in patients with metastatic neuroendocrine midgut tumors: a report from the PROMID Study Group. J Clin Oncol 2009; 27:4656-4663.
12 Pavel ME, Hainsworth JD, Baudin E, et al: RADIANT-2 Study Group. Everolimus plus octreotide long-acting repeatable for the treatment of advanced neuroendocrine tumours associated with carcinoid syndrome (RADIANT-2): a randomised, placebo-controlled, phase 3 study. Lancet 2011;378:20052012.

13 Kwekkeboom DJ, Krenning EP, Lebtahi R, et al: ENETS Consensus Guidelines for the Standards of Care in Neuroendocrine Tumors: peptide receptor radionuclide therapy with radiolabeled somatostatin analogs. Neuroendocrinology 2009;90:220-226.

14 Ruszniewski P, Rougier P, Roche A, et al: Hepatic arterial chemoembolization in patients with liver metastases of endocrine tumors. A prospective phase II study in 24 patients. Cancer 1993;71:2624-2630.

15 Marrache F, Vullierme MP, Roy C, et al: Arterial phase enhancement and body mass index are predictors of response to chemoembolisation for liver metastases of endocrine tumours. Br J Cancer 2007;96:49-55.

16 Dominguez S, Denys A, Madeira I, et al: Hepatic arterial chemoembolization with streptozotocin in patients with metastatic digestive endocrine tumours. Eur J Gastroenterol Hepatol 2000;12:151-157.

17 Roche A, Girish BV, de Baere T, et al: Transcatheter arterial chemoembolization as firstline treatment for hepatic metastases from endocrine tumors. Eur Radiol 2003;13:136140.

18 Diaco DS, Hajarizadeh H, Mueller CR, et al: Treatment of metastatic carcinoid tumors using multimodality therapy of octreotide acetate, intra-arterial chemotherapy, and hepatic arterial chemoembolization. Am J Surg 1995; 169:523-528.

19 Gupta S, Johnson MM, Murthy R, et al: Hepatic arterial embolization and chemoembolization for the treatment of patients with metastatic neuroendocrine tumors: variables affecting response rates and survival. Cancer 2005;104:1590-1602.

20 Kloppel G, Perren A, Heitz PU: The gastroenteropancreatic neuroendocrine cell system and its tumors: the WHO classification. Ann N Y Acad Sci 2004;1014:13-27.
21 Strosberg JR, Choi J, Cantor AB, et al: Selective hepatic artery embolization for treatment of patients with metastatic carcinoid and pancreatic endocrine tumors. Cancer Control 2006;13:72-78.

22 Osborne DA, Zervos EE, Strosberg J, et al: Improved outcome with cytoreduction versus embolization for symptomatic hepatic metastases of carcinoid and neuroendocrine tumors. Ann Surg Oncol 2006;13:572-581.

23 Bloomston M, Al-Saif O, Klemanski D, et al: Hepatic artery chemoembolization in 122 patients with metastatic carcinoid tumor: lessons learned. J Gastrointest Surg 2007;11: 264-271.

24 Ho AS, Picus J, Darcy MD, et al: Long-term outcome after chemoembolization and embolization of hepatic metastatic lesions from neuroendocrine tumors. Am J Roentgenol 2007;188:1201-1207.

25 Kamat PP, Gupta S, Ensor JE, et al: Hepatic arterial embolization and chemoembolization in the management of patients with large-volume liver metastases. Cardiovasc Intervent Radiol 2008;31:299-307.

26 Swärd C, Johanson V, Nieveen van Dijkum E, et al: Prolonged survival after hepatic artery embolization in patients with midgut carcinoid syndrome. Br J Surg 2009;96:517521.

27 Artinyan A, Nelson R, Soriano P, et al: Treatment response to transcatheter arterial embolization and chemoembolization in primary and metastatic tumors of the liver. $\mathrm{HPB}$ (Oxford) 2008;10:396-404.

28 Christante D, Pommier S, Givi B, et al: Hepatic artery chemoinfusion with chemoembolization for neuroendocrine cancer with progressive hepatic metastases despite octreotide therapy. Surgery 2008;144:885-894.

29 Brown DB, Pilgram TK, Darcy MD, et al: Hepatic arterial chemoembolization for hepatocellular carcinoma: comparison of survival rates with different embolic agents. J Vasc Interv Radiol 2005;16:1661-1666.

-30 Ruutiainen AT, Soulen MC, Tuite CM, et al: Chemoembolization and bland embolization of neuroendocrine tumor metastases to the liver. Vasc Interv Radiol 2007;18:847855

- 31 de Baere T, Deschamps F, Teriitheau C, et al: Transarterial chemoembolization of liver metastases from well differentiated gastroenteropancreatic endocrine tumors with doxorubicin-eluting beads: preliminary results. J Vasc Interv Radiol 2008;19:855-861. 